\title{
MODELLING THE PHYSICAL AND CHEMICAL EVOLUTION OF PAHS AND PAH-RELATED SPECIES IN ASTROPHYSICAL ENVIRONMENTS
}

\author{
J. Montillaud ${ }^{1,2}$, C. Joblin ${ }^{1,2}$ and D. Toublanc ${ }^{1,2}$
}

\begin{abstract}
An active carbon chemistry is observed at the border of photo-dissociation regions (PDRs), involving small hydrocarbons, polycyclic aromatic hydrocarbon (PAH) macromolecules and evaporating very small grains (VSGs). In this context, we aim at quantifying the physical and chemical evolution of PAHs (hydrogenation and charge states, aggregation, and complexation with heavy atoms) as a function of the local physical conditions (radiation field, temperature, density, abundances of atomic and molecular hydrogen, electrons and heavy atoms). We have developed a numerical model that follows the time dependency of the abundance and internal energy of each species. In this paper, we use this model to calculate the hydrogenation and charge states of coronene $\mathrm{C}_{24} \mathrm{H}_{12}$ as an interstellar $\mathrm{PAH}$ prototype. We take advantage of recent results on photodissociation and reaction rates and provide guidelines for future laboratory studies. Reaction rates of coronene-derived radical cations with $\mathrm{H}$ and $\mathrm{H}_{2}$ are found to be sufficiently constrained by experiments, whereas the absence of experimental data for neutral species is critical.
\end{abstract}

\section{Introduction}

During the two last decades, polycyclic aromatic hydrocarbons (PAHs) have been extensively studied as candidate carriers for the so-called aromatic infrared bands (AIBs). In order to test this hypothesis, models of increasing complexity and accuracy have been developed. Bakes \& Tielens (1994) described the charge state of PAHs and their contribution to the photoelectric heating. Allain et al. (1996a, 1996b) modelled the photodissociation rates of PAHs, taking into account the loss of $\mathrm{H}, \mathrm{H}_{2}$, and $\mathrm{C}_{2} \mathrm{H}_{2}$, and discussed their lifetime in the interstellar medium (ISM).

\footnotetext{
1 Université de Toulouse, UPS, CESR, 9 Av. du Colonel Roche, 31028 Toulouse Cedex 4, France

2 CNRS, UMR5187, 31028 Toulouse, France
} 
Le Page et al. (2001, 2003) further improved the description of the photophysical and chemical processes of PAHs and discussed the hydrogenation and charge states of PAHs in the diffuse ISM. Visser et al. (2007) built a model based on previous studies to describe the evolution of PAHs in protoplanetary disks.

The accuracy of the results provided by these models depends on how well photophysical and chemical processes can be quantified. Experimental data are often scarce and studies on fundamental processes are needed, which in general involve interdisciplinary collaborations between physicists, chemists, and astrophysicists.

In this paper, we analyse the question of the charge and hydrogenation states of coronene $\left(\mathrm{C}_{24} \mathrm{H}_{12}\right)$ taking advantage of experimental data obtained with the cold ion trap PIRENEA (Joblin et al. 2002a). Section 2 presents the model that was developed to perform this study. The chemical and physical data are discussed in Section 3. Results are given and discussed in Section 4.

\section{Presentation of the model}

The model includes all hydrogenation states from fully dehydrogenated to superhydrogenated coronene (0 to $24 \mathrm{H}$ ). We do not consider PAH anions since there is so far no clear evidence for their presence in the ISM and we are facing a lack of information in particular on their reactivity with hydrogen. The processes driving the evolution of charge and hydrogenation states are discussed in Section 3.

The time evolution of the species is computed using the rate equation formalism. Typical environments are PDRs in which the physics and chemistry are driven by UV photons. In order to describe as properly as possible the physical conditions in these PDRs, we use the Meudon PDR code (Le Petit et al. 2006) that provide a self-consistent set of physical parameters, as a function of depth inside the cloud.

\section{Charge and hydrogenation states}

\subsection{Charge balance}

In the scope of this work, we focus on the most external layers of PDRs. The $\mathrm{PAH}$ charge state is determined by the balance between the photoionization and the electron recombination with $\mathrm{PAH}$ cations. The photoionization rate for a given energy of the UV photon $h \nu$ is computed as the product of the absorption cross-section $\sigma_{a b s}(h \nu)$ by the photon flux $F_{\text {photon }}(h \nu)$ and by the ionization yield $Y_{\text {ion }}(h \nu)$. The values of $\sigma_{a b s}$ are from Malloci et al. (2004) and those of $Y_{\text {ion }}$ from Verstraete et al. (1990).

Electron recombination rates have been measured for a few small PAH cations, up to the pyrene cation $\mathrm{C}_{16} \mathrm{H}_{10}^{+}$(see Biennier et al. 2006). The classical law for a thin conducting disk (e.g. Verstraete et al. 1990) overestimates the rates for these small species, but is expected to be relevant for large PAH cations $\left(N_{C} \approx\right.$ 100). For species of intermediate size like coronene cation $\mathrm{C}_{24} \mathrm{H}_{12}^{+}$, we assume the recombination rate to be between the value of pyrene $\left(4.1 \times 10^{-6} \mathrm{~cm}^{3} \mathrm{~s}^{-1}\right.$ at 
$300 \mathrm{~K})$ and the value predicted by the classical law $\left(1.6 \times 10^{-5} \mathrm{~cm}^{3} \mathrm{~s}^{-1}\right.$ at $\left.300 \mathrm{~K}\right)$. We choose the mean value $k_{r e c}=1.0 \times 10^{-5} \mathrm{~cm}^{3} \mathrm{~s}^{-1}$ at $300 \mathrm{~K}$ and also consider the extreme cases $k_{r e c}=4.1 \times 10^{-6} \mathrm{~cm}^{3} \mathrm{~s}^{-1}$ and $k_{r e c}=1.6 \times 10^{-5} \mathrm{~cm}^{3} \mathrm{~s}^{-1}$. A dependence of $k_{r e c}$ on $T^{-1 / 2}$ is used (Bakes \& Tielens 1994).

For the sake of simplicity, the photoionization and electron recombination rates were supposed not to depend on the hydrogenation state.

\subsection{Reactivity of PAHs with hydrogen}

Only few studies have been dedicated to the reactivity of PAH cations with $\mathrm{H}$ or $\mathrm{H}_{2}$, and we found no data for the reactivity of neutral PAHs. We use no temperature dependence for neutral-ion reactions, and use reaction rates $k \propto T^{1 / 2}$ for neutral-neutral reactions. All the values hereafter are given at $300 \mathrm{~K}$ except when mentioned.

The reactivity of $\mathrm{PAH}$ cations with $\mathrm{H}$ has been studied experimentally for a few PAH cations (Bierbaum, see elsewhere in this volume) including coronene $\left(k_{+H}=1.4 \pm 0.7 \times 10^{-10} \mathrm{~cm}^{3} \mathrm{~s}^{-1}\right.$, Betts et al. 2006). We generalized the latter value to all coronene derivative species missing an even number of hydrogens, i.e. radical dehydrogenated cations. Cations missing an odd number of hydrogens are expected to be less reactive. This was demonstrated on naphthalene by Le Page et al. (1997), who measured $k_{+H}=1.9 \times 10^{-10}$ and $<5 \times 10^{-11} \mathrm{~cm}^{3} \mathrm{~s}^{-1}$ for $\mathrm{C}_{10} \mathrm{H}_{8}^{+}$and $\mathrm{C}_{10} \mathrm{H}_{7}^{+}$, respectively. Since no experimental data are available for closed-shell dehydrogenated coronene cations, we follow Le Page et al. (2001) and use a reaction rate of $k_{+H}=5 \times 10^{-11} \mathrm{~cm}^{3} \mathrm{~s}^{-1}$.

Betts et al. (2006) have evaluated an upper limit for the reactivity of $\mathrm{C}_{24} \mathrm{H}_{12}^{+}$ with $\mathrm{H}_{2}$ of $5 \times 10^{-13} \mathrm{~cm}^{3} \mathrm{~s}^{-1}$. We generalize this result to all radical dehydrogenated cations. Similarly, recent measurements using the cold ion trap PIRENEA (Joblin et al. 2002a) have shown no reactivity of $\mathrm{C}_{24} \mathrm{H}_{11}^{+}$with $\mathrm{H}_{2}$ at low pressure $\left(\sim 10^{-8}\right.$ mbar $)$ and temperature $(T<\approx 80 \mathrm{~K})$. An upper limit of $4 \times 10^{-12} \mathrm{~cm}^{3} \mathrm{~s}^{-1}$ was derived. Here again, we generalize this value to all closed-shell dehydrogenated coronene cations.

Superhydrogenated naphthalene and pyrene were measured not to react with $\mathrm{H}_{2}$, and to react slowly with $\mathrm{H}$ at rates around $\sim 10^{-12} \mathrm{~cm}^{3} \mathrm{~s}^{-1}$ (Snow et al. 1998). Hence we assume superhydrogenated coronene to react with $\mathrm{H}$ only, at a rate of $10^{-12} \mathrm{~cm}^{3} \mathrm{~s}^{-1}$. More informations concerning superhydrogenated PAHs can be found in Thrower et al. (see elsewhere in this volume).

\subsection{Photodissociation of coronene by hydrogen loss}

After absorption of a UV photon, a PAH can either dissociate or cool down radiatively. Allain et al. (1996a) showed that the relaxation cascade is dominated by IR photons. The IR emission rates were calculated in a microcanonical formalism (Joblin et al. 2002b).

Using the PIRENEA set-up, Joblin et al. studied the photodissociation of $\mathrm{C}_{24} \mathrm{H}_{p}^{+}$species $(p=[1,12])$ and derived dissociation rates as a function of the 
Table 1. Summary of the reaction rates $\left(\mathrm{cm}^{3} \mathrm{~s}^{-1}\right)$ at $300 \mathrm{~K}$ used in our model for coronene derivatives and their origin. The neutral counterparts are assumed to react neither with $\mathrm{H}$ nor with $\mathrm{H}_{2}$. (a) Betts et al. (2006); (b) Le Page et al. (2001); (c) Snow et al. (1998); (d) No detection with the PIRENEA set-up.

\begin{tabular}{lllllll}
\hline Species & $C_{24} H_{n}^{+}$(even $\left.n \leq 12\right)$ & $C_{24} H_{n}^{+}$(odd $\left.n<12\right)$ & \multicolumn{2}{c}{$C_{24} H_{n}^{+}(n>12)$} \\
\hline$+H$ & $1.4 \times 10^{-10}$ & (a) & $5.0 \times 10^{-11}$ & (b) & $1.0 \times 10^{-12}$ & (c) \\
$+H_{2}$ & $<5 \times 10^{-13}$ & (a) & $<4 \times 10^{-12}$ & (d) & - & (b) \\
$+e^{-}$ & \multicolumn{5}{c}{$1.0 \times 10^{-5}$ (extrapolation) } \\
\hline
\end{tabular}

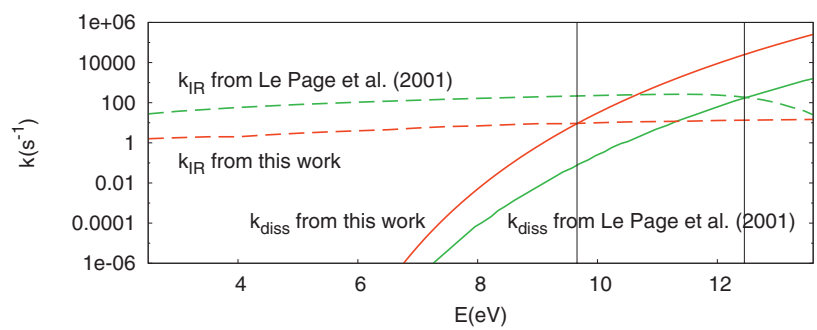

Fig. 1. Comparison of the IR cooling (dashed lines) and photodissociation (solid lines) rates from Le Page et al. (2001) (green lines) and from this work (red lines) for the coronene cation $\mathrm{C}_{24} \mathrm{H}_{12}^{+}$, as a function of its internal energy. Vertical lines indicate the threshold energy above which the dissociation is faster than the IR emission.

internal energy (Joblin et al. 2010a). Only H loss was observed for these species, the destruction of the carbon skeleton occuring only after complete dehydrogenation $\left(\mathrm{C}_{24}^{+}\right.$; Joblin et al. 2003; Joblin et al. 2010b). The reaction rates derived by Joblin et al. differ significantly from the values from Le Page et al. (2001; cf. Fig. 1).

\section{Results}

Using our numerical model, we compute the hydrogenation and charge states of coronene for the northern PDR in NGC 7023. For the incident radiation field, we used the stellar spectrum from the Kurucz library (Kurucz 1991) for a star with a temperature of $15000 \mathrm{~K}$ and an integrated UV intensity of 2600 Habing. The density was set to $n_{\mathrm{H}}=2 \times 10^{4} \mathrm{~cm}^{-3}$ (Gerin et al. 1998).

General behaviour. The hydrogenation state of coronene adopts four possible regimes: (1) totally dehydrogenated $\left(\mathrm{C}_{24}^{0 /+}\right.$ dominates $),(2)$ partially dehydrogenated with a roughly equivalent abundance for each $\mathrm{C}_{24} \mathrm{H}_{p}^{+}$, species $(p=$ $[1,11])$, (3) normally hydrogenated $\left(\mathrm{C}_{24} \mathrm{H}_{12}^{0 /+}\right.$ dominates $)$ and (4) superhydrogenated $\left(\mathrm{C}_{24} \mathrm{H}_{13}^{0 /+}\right.$ dominates).

Hydrogenation and charge state of coronene in NGC 7023. As shown in Figure 2, coronene is found to be essentially neutral throughout the PDR. Despite 
the high intensity of the radiation field, PAHs are ionized only in the first layers of the cloud. The totally dehydrogenated species, $\mathrm{C}_{24}$, dominates inside the PDR up to a depth of $A_{V} \approx 5$, where $G_{0} \approx 20$ in Habing units. For equivalent conditions, i.e. $G_{0} / n_{\mathrm{H}} \approx 1 / 1000$, Le Page et al. found the normal hydrogen coverage to dominate. This difference is consistent with our model using larger photodissociation rates, and lower reaction rates with $\mathrm{H}_{2}$.

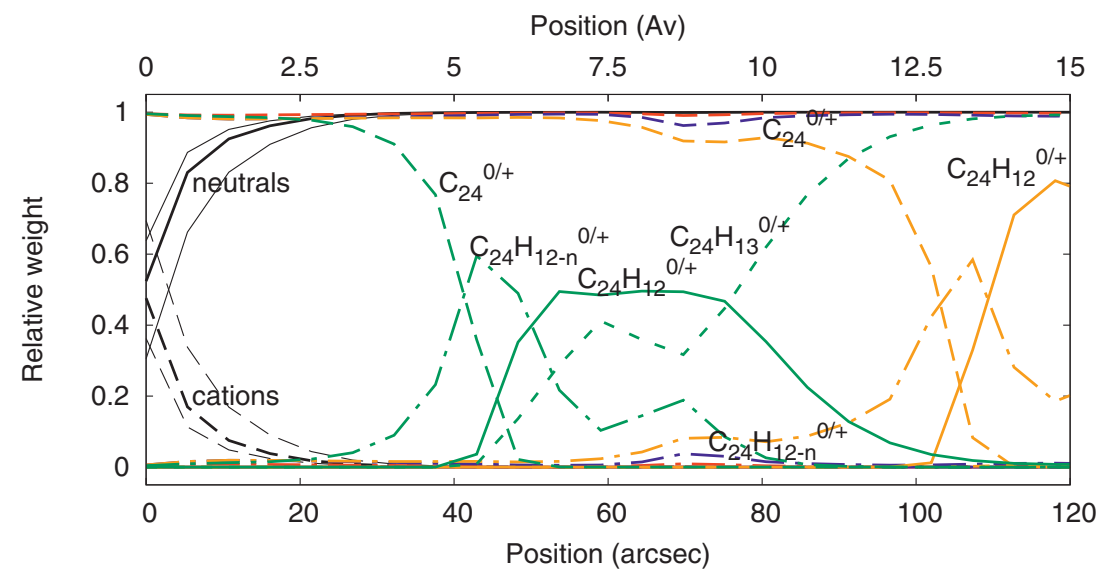

Fig. 2. Evolution of the charge and hydrogenation states of coronene through the northern PDR of the reflection nebula NGC 7023. Black lines: evolution of the abundances of cations (dashed lines) and neutrals (solid lines), for different electron recombination rates (thick lines: $k_{r e c}(300 \mathrm{~K})=1.0 \times 10^{-5} \mathrm{~cm}^{3} \mathrm{~s}^{-1}$; thin lines: $k_{r e c}(300 \mathrm{~K})=0.4$ and $\left.1.6 \times 10^{-5} \mathrm{~cm}^{3} \mathrm{~s}^{-1}\right)$. Color lines: abundances of $\mathrm{C}_{24}^{0 /+}$ (dashed lines), partially dehydrogenated species $\mathrm{C}_{24} \mathrm{H}_{12-n}^{+}$with $\mathrm{n}=[1,11]$ (dot-dashed lines), $\mathrm{C}_{24} \mathrm{H}_{12}^{+}$(solid lines) and $\mathrm{C}_{24} \mathrm{H}_{13}^{+}$(dotted lines) derived with (i) our standard model (red), (ii) maximum values for reactivity between cations and $\mathrm{H}_{2}$ (blue), (iii) reaction rates of neutral radicals with $\mathrm{H}$ set to $1.4 \times 10^{-11} \mathrm{~cm}^{3} \mathrm{~s}^{-1}$ at $300 \mathrm{~K}$ (orange) and (iv) reaction rates of neutrals with $\mathrm{H}$ set at $300 \mathrm{~K}$ to $1.4 \times 10^{-11} \mathrm{~cm}^{3} \mathrm{~s}^{-1}$ (radicals) and $5.0 \times 10^{-12} \mathrm{~cm}^{3} \mathrm{~s}^{-1}$ (closed-shells) (green).

Influence of the uncertainties. The uncertainty on the electron recombination rate affects the ionization fraction to at most a factor of 2 . Tuning the reaction rates of $\mathrm{PAH}$ cations with $\mathrm{H}_{2}$ from their upper limit to zero does not change significantly the results. The abundances are also not strongly affected by the experimental uncertainty of $50 \%$ on the reactivity of $\mathrm{C}_{24} \mathrm{H}_{12}^{+}$with $\mathrm{H}$. On the contrary, tuning the reactivity of neutral PAHs, which was set to 0 in our standard model, leads to substantial changes. If neutral radicals $\mathrm{C}_{24} \mathrm{H}_{2 n-1}$ react with $\mathrm{H}$ at a rate at $300 \mathrm{~K}$ ten times lower than cation radicals, the hydrogenation state differs only deep in the cloud $\left(A_{V}>10\right)$, where PAHs are very likely in solid phase. However, if neutral closed-shell species $\mathrm{C}_{24} \mathrm{H}_{2 n}$ are also considered to react with $\mathrm{H}$, with a rate at $300 \mathrm{~K}$ ten times lower than for closed-shell cations, then $\mathrm{C}_{24} \mathrm{H}_{12}$ dominates between $A_{V}=6$ and 9 , while $\mathrm{C}_{24} \mathrm{H}_{13}$ dominates deeper. 


\section{Conclusion}

We present the first results of a model dedicated to the study of the evolution of PAHs in astrophysical environments. We examine the case of coronene hydrogenation and charge states, in the northern PDR of NGC 7023, using the latest available physical and chemical data. Coronene was found to be much more dehydrogenated than calculated in previous studies. Reaction rates of coronene-derived radical cations with $\mathrm{H}$ and $\mathrm{H}_{2}$ were found to be sufficiently constrained by experiments, whereas the absence of experimental data for the reactivity of neutral species appears to be critical. A first theoretical study is presented elsewhere in this volume (Thrower et al.).

\section{References}

Allain, T., Leach, S., \& Sedlmayr, E., 1996a, A\&A, 305, 602

Allain, T., Leach, S., \& Sedlmayr, E., 1996b, A\&A, 305, 616

Bakes, E.L.O., \& Tielens, A.G.G.M., 1994, ApJ, 427, 822

Betts, N.B., Stepanovic, M., Snow, T.P., \& Bierbaum, V.M., 2006, ApJ, 651, L129

Biennier, L., Alsayed-Ali, M., Foutel-Richard, A., et al., 2006, Faraday Discussions, 133, 289

Draine, B.T., \& Sutin, B., 1987, ApJ, 320, 803

Gerin, M., Phillips, T., Keene, J., Betz, A.L., \& Boreiko, R.T., 1998, ApJ, 500, 329

Joblin, C., Pech, C., Armengaud, M., Frabel, P., \& Boissel, P., 2002a, EAS Publication Series, 4,73

Joblin, C., Toublanc, D., Boissel, P., \& Tielens, A.G.G.M., 2002b, Mol. Phys., 100, 3595

Joblin, C., 2003, in SF2A-2003, ed. F. Combes et al. (EDP-Sciences), 175

Joblin C., Toublanc D., Pech C., Armengaud M., Frabel P., \& Boissel P., 2010a, in preparation

Joblin C., Armengaud M., Frabel P., \& Boissel P., 2010b, in preparation

Jochims, H.W., Ruhl, E., Baumgartel, H., Tobita, S., \& Leach, S., 1994, ApJ, 420, 307

Kurucz, R.L., 1991, BAAS, 23, 1047

Leger, A., d'Hendecourt, L., Boissel, P., \& Desert, F.X., 1989, A\&A, 213, 351

Le Page, V., Keheyan, Y., Bierbaum, V.M., \& Snow, T.P., 1997, JACS, 119, 35

Le Page, V., Keheyan, Y., Snow, T.P., \& Bierbaum, V.M., 1999a, IJMS, 185, 949

Le Page, V., Keheyan, Y., Snow, T.P., \& Bierbaum, V.M., 1999b, JACS, 121, 9435

Le Page, V., Snow, T.P., \& Bierbaum, V.M., 2001, ApJS, 132, 233

Le Page, V., Snow, T.P., \& Bierbaum, V.M., 2003, ApJ, 584, 316

Le Petit, F., Nehme, C., Le Bourlot, J., \& Roueff, E., 2006, ApJS, 164, 506

Malloci, G., Mulas, G., \& Joblin, C., 2004, A\&A, 426, 105

Snow, T.P., Le Page, V., Keheyan, Y., \& Bierbaum, V.M., 1998, Nature, 391, 259

Verstraete, L., Leger, A., D’Hendecourt, L., Defourneau, D., \& Dutuit, O., 1990, A\&A, 237,436

Visser, R., Geers, V.C., Dullemond, C.P., et al., 2007, A\&A, 466, 229 